\title{
DETECTION OF RESISTIVE TRANSITIONS IN LHC SUPERCONDUCTING COMPONENTS
}

\author{
R. Denz, F. Rodriguez-Mateos, CERN, Geneva, Switzerland
}

\begin{abstract}
The LHC has entered the construction phase [1]. It will incorporate a large number of superconducting components like magnets, current leads and busbars. All these components require protection means in case of a transition from the superconducting to the resistive state, the so-called quench. Key elements in the protection system [2] are electronic quench detectors, which have to be able to identify a quench in any state of the powering cycle of the accelerator. According to the different properties and characteristics of the superconducting elements and circuits, a set of quench detectors adapted to their specific tasks has been developed.
\end{abstract}

\section{INTRODUCTION}

\subsection{General layout and parameters}

The general layout of a quench detector can be subdivided into 4 functional blocks (see Fig. 1): an amplifying and buffering input stage, a comparator, a time discriminator and a driver for transmitting the quench status signal. In addition, there is a data acquisition and test system integrated into each quench detector, which will be used for diagnostics, power permit and post mortem analysis.

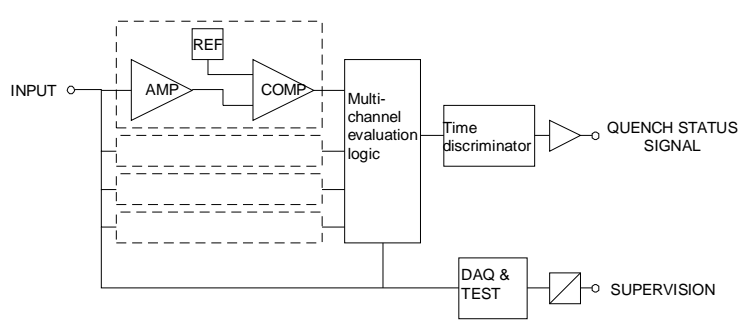

Figure 1: General quench detector layout.

The main parameters of a quench detector are the threshold voltage $U_{T h}$ and the time delay of the time discriminator $t_{\text {Dis }}$ (see Table 1 ). The time discriminator is necessary to increase the noise immunity of a quench detector, i.e. reduce the faulty detection rate. In case of a quench, $U_{T h}$ is reached after a certain time, which depends on the physical properties of the superconducting device, its temperature, circuit characteristics and operating current. The quench detector itself needs time to process the acquired signal and finally the activation of the protection system will also require some time. The sum of all these individual delays, the initial temperature and the operating current will determine the amount of energy dissipated in the protected component, the so-called quench load [3]. Taking into consideration the safe operating limits of the superconducting components, the maximum quench load and the detector parameters can be determined.

Table 1: General Parameters of LHC Quench Detectors

\begin{tabular}{|l|l|l|l|}
\hline Component & Qty & $\mathbf{U}_{\text {Th }}[\mathbf{m V}]$ & $\mathbf{T}_{\text {Dis }}[\mathbf{m s}]$ \\
\hline $\begin{array}{l}\text { Main dipoles \& } \\
\text { lattice quadrupoles }\end{array}$ & 2050 & 200 & 10 \\
\hline $\begin{array}{l}\text { Current leads: } \\
\text { resistive part }\end{array}$ & 650 & 150 & 100 \\
\hline $\begin{array}{l}\text { Current leads: } \\
\text { superconducting } \\
\text { part }\end{array}$ & 20 & 200 & 10 \\
\hline $\begin{array}{l}\text { Insertion region } \\
\text { magnets }\end{array}$ & 190 & 100 & 10 \\
\hline $\begin{array}{l}\text { Corrector magnet } \\
\text { circuits }\end{array}$ & 430 & 1000 & 5000 \\
\hline 13kA busbars & 24 & & \\
\hline
\end{tabular}

\subsection{Galvanic isolation and electromagnetic compatibility}

The quench detector must provide galvanic isolation between input and output as well as to ground according to the required withstand levels [4]. All isolating components of the quench detector such as the power supply, digital isolators (pulse transformers) and relays exhibit voltage withstand levels of $2.5 \mathrm{kV}$.

The electrical safety and electromagnetic compatibility of the quench detector power supplies must be certified according to EU norms (CE mark).

\subsection{Interfaces}

All quench detectors issue information to the machine protection system and to the accelerator supervision system. All information relevant for the accelerator supervision system, post mortem analysis, test mode enabling and power permit will be transmitted via fieldbuses and Ethernet links. The quench status signal, which changes to low any time a quench occurs, will be transmitted via hardwired links to the machine protection system. In the case of the main dipole and lattice quadrupoles, this link is realized using of a current loop.

\subsection{Location}

The quench detectors for the main dipoles and lattice quadrupoles are located in racks under the main dipoles inside the LHC tunnel. These racks house also the quench 
heater power supplies. One part of the quench detection system for the 13kA superconducting busbars will be installed in the alcoves, the rest, as well as all other quench detectors, in the LHC underground areas close to the corresponding power converters.

\section{MAIN DIPOLES AND LATTICE QUADRUPOLES}

\subsection{Working principle}

A quench in one of the main dipoles is detected by a floating bridge detector, which continuously compares the voltages of the two apertures. The two magnet apertures, which both have equal inductances, and two balancing resistors form the bridge (see Fig. 2). These resistors will also be used for over-current protection of the instrumentation wires. The same kind of detector compares the voltage drops across two different poles of a lattice quadrupole magnet, where the two apertures are powered separately. In case of a quench, the floating bridge will be no longer balanced and the detector will activate directly the associated quench heater power supplies. At the same time a safety relay will open the respective current loop, which triggers the machine protection system. It is noteworthy that a detection of a quench in any aperture of a quadrupole will energize all quench heater strips of the magnet.

\subsection{Reliability}

In order to increase its reliability, the quench detector is based on multi-channel evaluation, which used originally a " 2 out of 3" scheme. Recent calculations [5] however show that a "2 out of 4" scheme is more advantageous with respect to accelerator down time. In order to ensure the integrity of the protected magnet a loss of two parallel instrumentation wires, a power failure of the detector or at the current loop, and an earth fault of the current loop will be treated as a quench.

\subsection{Data acquisition and test mode}

The quench detector is equipped with a small dataacquisition system providing information on the voltage across the magnet apertures, the output of the bridge as well as a number of status flags. The system uses a circular buffer; in case of a quench its content will be frozen. A test mode unit enables the generation of test signals for the quench detector. This type of test serves also to verify the functionality of the associated quench heater power supplies by discharging them into the cold but un-powered magnet. It is foreseen to perform this kind of test about two times a year.

\subsection{Failure of the energy extraction system}

The protection schemes of the main dipole and quadrupole circuits are based on active energy extraction [6]. In the very unlikely event that this system is not able to open the circuit breakers in case of a quench, it will request to energize the quench heaters in selected magnets. The provoked quenches will help to re-establish the original discharge time constant of the circuit thus avoiding major damage to the superconducting magnet chain. This request will be processed automatically by the energy extraction system and by the quench detectors, which will fire the corresponding quench heater power supplies.

\subsection{Radiation tolerance}

As the quench detectors will be installed in the LHC tunnel under the main dipoles, they have to be qualified also with respect to the necessary radiation withstand levels. A dedicated test program has already been performed over the last two years giving promising results for total integrated doses up to 500Gy [7].

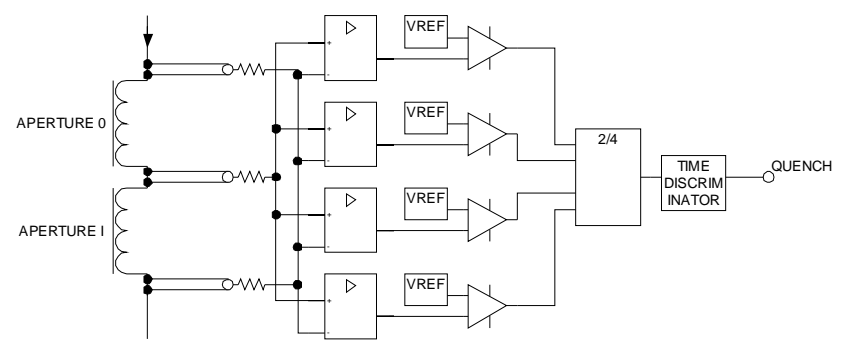

Figure 2: Floating bridge type quench detector for main dipole.

\section{SUPERCONDUCTING CURRENT LEADS}

Each of the 650 pairs of HTS-based superconducting current leads [8] is protected by a specially designed detector, which supervises the resistive part of the leads as well as the superconducting part. Like the detectors for the main magnets the input stage is based on analog circuitry and will use a 2 out of 4 multi-channel evaluation scheme. In order to cope with the relatively low threshold voltage value of the superconducting part, active offset voltage compensation will be implemented in the detector input stage.

\section{CORRECTOR MAGNETS}

Differential voltage detectors with dedicated current sensors will protect all superconducting circuits up to a nominal current of 600A. The differential voltage is picked up at the cold ends of the superconducting current leads. The detector is equipped with a dedicated current sensor enabling it to measure the current and calculate the derivative. The current transducer is of the Hall type and has a precision and linearity of $1 \%$, which is acceptable for the error budget of the detector. The hardware is based on a digital signal processor (DSP). The DSP is processing the incoming signals and calculates the resistive voltage drop with the help of preloaded inductance tables thus providing active compensation of 
the inductive voltages. These tables will be generated and updated during special calibration cycles. Active filtering algorithms, implemented in the DSP code, will be used to reduce the electromagnetic susceptibility.

\section{INSERTION REGION MAGNETS}

These magnets have nominal currents up to $7.5 \mathrm{kA}$. For this reason a dedicated current sensor for the quench detector is not feasible for space and cost reasons. Voltage taps connected to the midpoints of the circuits and the cold ends of the current leads however offer the possibility of using a floating bridge type quench detector. In this type of detector the superconducting busbars will also be part of the bridge. As for the corrector magnet circuits the quench detector is based on a DSP, which allows implementing digital filters and compensation of inductances. In case of a quench the detector will transmit the quench status signal and fire the corresponding quench heater power supplies.

The same type of detector will also be used to protect the inner triplet quadrupole magnets. not necessary, as the magnets incorporate their own quench detectors.

In case additional superconducting links will be installed in LHC, the same type of quench detector may be used for their protection.

\section{STATUS AND CONCLUSIONS}

Prototype and pre-series devices of almost all of these different types of detectors have been built and tested. Most of them were installed in the String II [9] experiment, which is a full-scale mock-up of the LHC standard cell. At the time of the conference, the commissioning of STRING II has started and first powering is expected soon. STRING II will be used for extensive tests of all type of quench detectors. In addition it will be possible to run STRING II in special configurations, e.g. to test the protection scheme for insertion region magnets. At the same time device and component qualification, which includes radiation tests, continues. The final technical specifications of the quench detectors will be available beginning of 2002 .

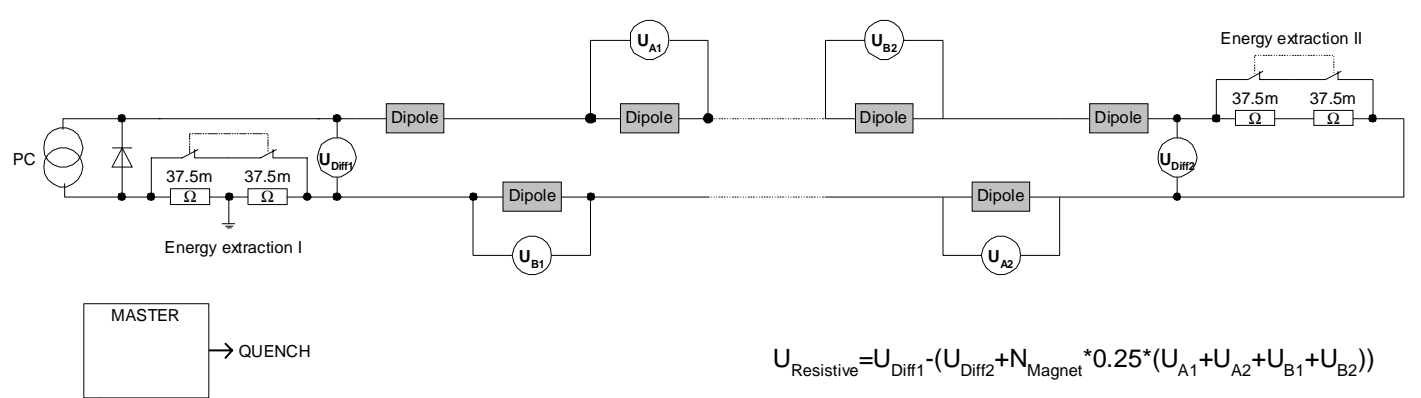

Figure 3: Protection of 13kA Superconducting Busbars.

\section{SUPERCONDUCTING BUSBARS}

Unlike the superconducting busbars of the corrector and insertion region magnets, which are included in the protection of the attached magnets, the protection of the $13 \mathrm{kA}$ main busbars is a special case requiring a dedicated quench detector (see Fig. 3). This quench detector is builtup with a cluster of digital voltmeters measuring the differential voltage drops at the cold ends of the current leads as well as across selected magnets. All voltmeters communicate their signals to a master device, which is calculating the resistive voltage of the superconducting busbars. The hardware of the master device consists of an industrial PC running a real time operation system. This approach is especially necessary for the protection of the main dipole circuit, which has a total inductance of about $16 \mathrm{H}$ and two energy extraction systems.

The design of the quench detector does not allow detecting quenches in the magnets themselves, which is

\section{REFERENCES}

[1] R. Ostojic, "Status of the LHC", this conference.

[2] K. Dahlerup-Petersen, R. Denz, J. L. Gomez-Costa, D. Hagedorn, P. Proudlock, F. Rodriguez-Mateos, R. Schmidt and F. Sonnemann, "The Protection System for the Superconducting Elements of the Large Hadron Collider at CERN", LHC Project Report 283, PAC'99, New York, USA, March 29 - April 2, 1999.

[3] F. Rodriguez-Mateos, F. Sonnemann, "Quench Heater Studies for the LHC Magnets", this conference.

[4] F. Rodriguez-Mateos, "Voltage Withstand Levels for Electrical Insulation Tests", LHC-PM-ES-0001, 1999.

[5] A. Vergara, private communication.

[6] K. Dahlerup-Petersen, A. Medvedko, A. Erokhin, B. Kazmin, V. Sytchev, L. Vassiliev, "Energy Extraction in the CERN Large Hadron Collider, -a Project Overview", PPPS2001, Las Vegas June 2001.

[7] R. Denz, F. Rodriguez-Mateos and A. Vergara, "Irradiation Tests on COTS used in the Protection System of LHC Superconducting Elements", RADECS 2000, Louvain-la-Neuve (Belgium), September 2000.

[8] A.Ballarino, "Applications of High Temperature Superconductors to Accelerators", EPAC'00, Vienna (Austria) June 2000.

[9] R. Saban, "The Commissioning of the LHC Test String 2", this conference. 